Data Article

\title{
Survey data regarding perceived air quality in Australia, Brazil, China, Ghana, India, Iran, Italy, Norway, South Africa, United States

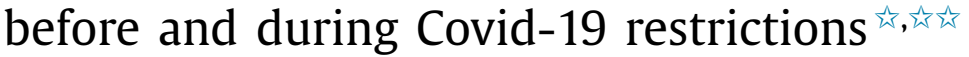

\author{
Diego Maria Barbieri ${ }^{\mathrm{a}, *}$, Baowen Lou ${ }^{\mathrm{b}}$, Marco Passavanti ${ }^{\mathrm{c}}$,
} Cang Hui ${ }^{\mathrm{d}}$, Daniela Antunes Lessa ${ }^{\mathrm{e}}$, Brij Maharaj ${ }^{\mathrm{f}}$, Arunabha Banerjee ${ }^{g}$, Fusong Wang ${ }^{\mathrm{h}}$, Kevin Chang ${ }^{\mathrm{i}}$, Bhaven Naik ${ }^{\mathrm{j}}$, Lei Yu ${ }^{\mathrm{k}}$, Zhuangzhuang Liu, Gaurav Sikka ${ }^{\mathrm{m}}$, Andrew Tucker ${ }^{\mathrm{n}}$, Ali Foroutan Mirhosseini ${ }^{\circ}$, Sahra Naserip, Yaning Qiao ${ }^{q}$, Akshay Gupta ${ }^{\mathrm{r}}$, Montasir Abbass ${ }^{\mathrm{s}}$, Kevin Fang ${ }^{\mathrm{t}}$, Navid Ghasemi ${ }^{\mathrm{u}}$, Prince Peprah ${ }^{v}$, Shubham Goswami ${ }^{w}$, Amir Hessami ${ }^{\mathrm{x}}$, Nithin Agarwaly, Louisa Lam ${ }^{z}$, Solomon Adomako ${ }^{\$}$

\footnotetext{
a Norwegian University of Science and Technology, Department of Civil and Environmental Engineering. Høgskoleringen 7A, Trondheim, 7491, Trøndelag, Norway

b Chang'an University, School of Highway, Nan Er Huan Road (Mid-section), Xi'an, 710064, Shaanxi, China ${ }^{c}$ Italian Society of Cognitive Behavioural Therapy (CBT-Italy), Guastalla St. 2, Carpi 4012, Emilia-Romagna, Italy ${ }^{\mathrm{d}}$ Centre for Invasion Biology, Department of Mathematical Sciences, Stellenbosch University, Matieland, 7602, South Africa.

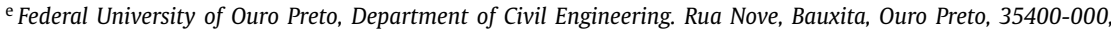
Minas Gerais, Brazil

${ }^{\mathrm{f}}$ University of KwaZulu-Natal, Department of Geography. Howard College City, Durban, 4000, KwaZulu, South Africa ${ }^{\mathrm{g}}$ Indian Institute of Technology Guwahati, Department of Civil Engineering. IIT Guwahati, Guwahati, 781039, Assam, India

${ }^{\mathrm{h}}$ State Key Laboratory of Silicate Materials for Architectures, Wuhan University of Technology. Luoshi road 122, Wuhan, 430070, Hubei, China

i University of Idaho, Department of Civil and Environmental Engineering. 875 Perimeter Drive, Mailstop 1022, Moscow, 83844, Idaho, United States

j Ohio University, Department of Civil Engineering/Russ College of Engineering E Technology. 28 W. Green Drive, Athens, 45701, Ohio, United States

${ }^{\mathrm{k}}$ Sun Yat-sen University, School of Civil Engineering. Xingang Xi Road 135, Guangzhou, 510275, Guangdong, China

'Chang'an University, School of Highway. Nan Er Huan Road (Mid-section), Xi'an, 710064, Shaanxi, China

${ }^{\mathrm{m}}$ Lalit Narayan Mithila University, Department of Geography. Darbhanga, 846004, Bihar, India

${ }^{\mathrm{n}}$ University of Connecticut, Connecticut Transportation Safety Research Center. 270 Middle Turnpike, Unit 5202

Longley Building, Storrs, 06269, Connecticut, United States

${ }^{\circ}$ Norwegian University of Science and Technology, Department of Civil and Environmental Engineering.

Høgskoleringen 7A, Trondheim, 7491, Trøndelag, Norway

p Bam University of Medical Sciences, School of Medicine. Bam, 76615-336, Kerman, Iran
} 
${ }^{\mathrm{q}}$ China University of Mining and Technology, School of Mechanics and Civil Engineering. Daxue Road 1, Xuzhou, 22116, Jiangsu, China

${ }^{\mathrm{r}}$ Indian Institute of Technology Roorkee, Department of Civil Engineering, Transportation Engineering Group, 321-AEB, Roorkee, 247667, Uttarakhand, India

${ }^{\mathrm{s}}$ Virginia Tech, Department of Civil and Environmental Engineering. 301-D3 Patton Hall, Blacksburg, 24061, Virginia, United States

${ }^{\mathrm{t}}$ Sonoma State University, Department of Geography, Environment, and Planning, 1801 East Cotati Avenue, Rohnert Park, 94928, California, United States

"University of Bologna, Department of Civil Chemical Environmental and Materials Engineering. Viale del Risorgimento, 2, Bologna, 40136, Emilia-Romagna, Italy

v University of New South Wales, Department of Social Policy Research Centre, John Goodsell Building, Kensington, Sydney, 2052, New South Wales, Australia

${ }^{\mathrm{w}}$ Indian Institute of Science Bangalore, Department of Civil Engineering, C V Raman Avenue, Bangalore, 560012, Karnataka, India

${ }^{x}$ Texas AEM University - Kingsville, Department of Civil and Architectural Engineering, 917 W. Ave B, Kingsville, 78363, Texas, United States

y University of Florida, Department of Civil \& Coastal Engineering, 2100 NE Waldo Rd., Sta 106, Gainesville, 32609, Florida, United States

${ }^{\mathrm{z}}$ Federation University Australia, School of Nursing and Healthcare Professions, 72-100 Clyde Rd, Berwick, 3806, Victoria, Australia

\$University of Agder, Department of Engineering and Science, Jon Lilletuns vei 9, Grimstad, 4879, Agder, Norway

\section{A R T I C L E I N F O}

Article history:

Received 14 July 2020

Accepted 6 August 2020

Available online 13 August 2020

Keywords:

Survey data

COVID-19

Environmental pollution

Air quality

Psychometric perception

\section{A B S T R A C T}

The dataset deals with the air quality perceived by citizens before and during the enforcement of COVID-19 restrictions in ten countries around the world: Australia, Brazil, China, Ghana, India, Iran, Italy, Norway, South Africa and the United States. An online survey conveniently translated into Chinese, English, Italian, Norwegian, Persian, Portuguese collected information regarding the perceived quality of air pollution according to a Likert scale. The questionnaire was distributed between 11-05-2020 and 31-05-2020 and 9394 respondents took part. Both the survey and the dataset (stored in a Microsoft Excel Worksheet) are available in a public repository. The collected data offer the people's subjective perspectives related to the objective improvement in air quality occurred during the COVID-19 restrictions. Furthermore, the dataset can be used for research studies involving the reduction in air pollution as experienced, to a different extent, by populations of all the ten countries.

(c) 2020 The Author(s). Published by Elsevier Inc. This is an open access article under the CC BY license. (http://creativecommons.org/licenses/by/4.0/)

\footnotetext{
Declarations of interest: none

计的 Initial submission date: 14/07/2020

* Corresponding author.
}

E-mail addresses: diego.barbieri@ntnu.no (D.M. Barbieri), loubaowen@chd.edu.cn (B. Lou), m.passavanti@campus.unimib.it (M. Passavanti), chui@sun.ac.za (C. Hui), daniela.lessa@ufop.edu.br (D.A. Lessa), maharajb@ukzn.ac.za (B. Maharaj), wangfs@whut.edu.cn (F. Wang), kchang@uidaho.edu (K. Chang), naik@ohio.edu (B. Naik), yulei26@mail2.sysu.edu.cn (L. Yu), zzliu@chd.edu.cn (Z. Liu), andrew.tucker@uconn.edu (A. Tucker), ali.mirhosseini@ntnu.no (A.F. Mirhosseini), yaning.qiao@cumt.edu.cn (Y. Qiao), akshay_g@ce.iitr.ac.in (A. Gupta), abbas@vt.edu (M. Abbas), fangk@sonoma.edu (K. Fang), navid.ghasemi3@unibo.it (N. Ghasemi), gshubham@iisc.ac.in (S. Goswami), hessami_amir@tamu.edu (A. Hessami), nithin.agarwal@ufl.edu (N. Agarwal), 1.lam@federation.edu.au (L. Lam), solomon.adomako@uia.no (S. Adomako). 


\section{Specification table}

\begin{tabular}{|c|c|}
\hline Subject & Social Sciences \\
\hline Specific subject area & Health psychology, Perceived air pollution \\
\hline Type of data & Primary data, Table \\
\hline How data were acquired & $\begin{array}{l}\text { The data were collected by an online survey hosted on two platforms: } \\
\text { Google Forms (English, Italian, Norwegian, Persian, Portuguese } \\
\text { versions) and WenJuanXing (Chinese version). An English copy is } \\
\text { available in the data repository. The survey was distributed by means } \\
\text { of professional and social networks }\end{array}$ \\
\hline Data format & Raw Analyzed \\
\hline Parameters for data collection & $\begin{array}{l}\text { The survey data were obtained from } 9394 \text { respondents older than } 18 \\
\text { years old having internet access }\end{array}$ \\
\hline Description of data collection & $\begin{array}{l}\text { The online survey was distributed using a combination of purposive } \\
\text { and snowball techniques }\end{array}$ \\
\hline Data source location & $\begin{array}{l}\text { Countries: Australia, Brazil, China, Ghana, India, Iran, Italy, Norway, } \\
\text { South Africa and the United States }\end{array}$ \\
\hline Data accessibility & $\begin{array}{l}\text { Dataset is uploaded on Mendeley Data } \\
\text { Repository name: } \\
\text { Perceived air pollution in Australia, Brazil, China, Ghana, India, Iran, } \\
\text { Italy, Norway, South Africa, USA before and during COVID-19 } \\
\text { restrictions } \\
\text { Data identification number: } \\
\text { DOI: } 10.17632 / \text { fb38h4tyzn. } 2 \\
\text { Direct URL to data: https://data.mendeley.com/datasets/fb38h4tyzn/2 }\end{array}$ \\
\hline
\end{tabular}

Value of the data

- The data are related to the perception of air quality and air pollution during the COVID19 restrictions as experienced by a large pool comprising 9394 respondents located in ten countries on six continents

- The data can be useful for researchers dealing with the environmental and tropospheric changes occurring during the COVID-19 restrictions

- The data can be used to assess the relationship between the perceived and the quantified change in air quality and air pollution during the COVID-19 restrictions

- The data can be of interest to both citizens and policymakers to realise the tremendous lesson learned during COVID-19, being air quality a key indicator for sustainable development

\section{Data description}

The dataset provides information regarding the quantity of air pollution perceived before and during the restrictions enforced in ten countries around the world as a consequence of the COVID-19 pandemic: Australia, Brazil, China, Ghana, India, Iran, Italy, Norway, South Africa and the United States (also referred to as AU, BR, CH, GH, IN, IR, IT, NO, ZA and USA, respectively). The dataset is stored in a public repository as Microsoft Excel Worksheet [1]. The total amount of the respondents who joined the survey is 9394 , their geographical distribution is reported in Table 1. Information regarding gender and age are reported in Fig. 1 with box-and-whisker plots: overall, the largest portion of the surveyed population is composed of young and middleaged individuals. Furthermore, the participants have high education (Fig. 2). The two questions of the survey are "How do you regard the amount of air pollution before the epidemic?" and "How do you regard the amount of air pollution during the restrictions?": the respondents expressed their opinions according to a 7-point Likert scale varying from "extremely low/absent air pollution" to "extremely high air pollution". The responses pertaining to before and during the applications of the COVID-19 restrictions are reported in Fig. 3a and Fig. 3b, respectively. 
Table 1

Geographical distribution of survey respondents.

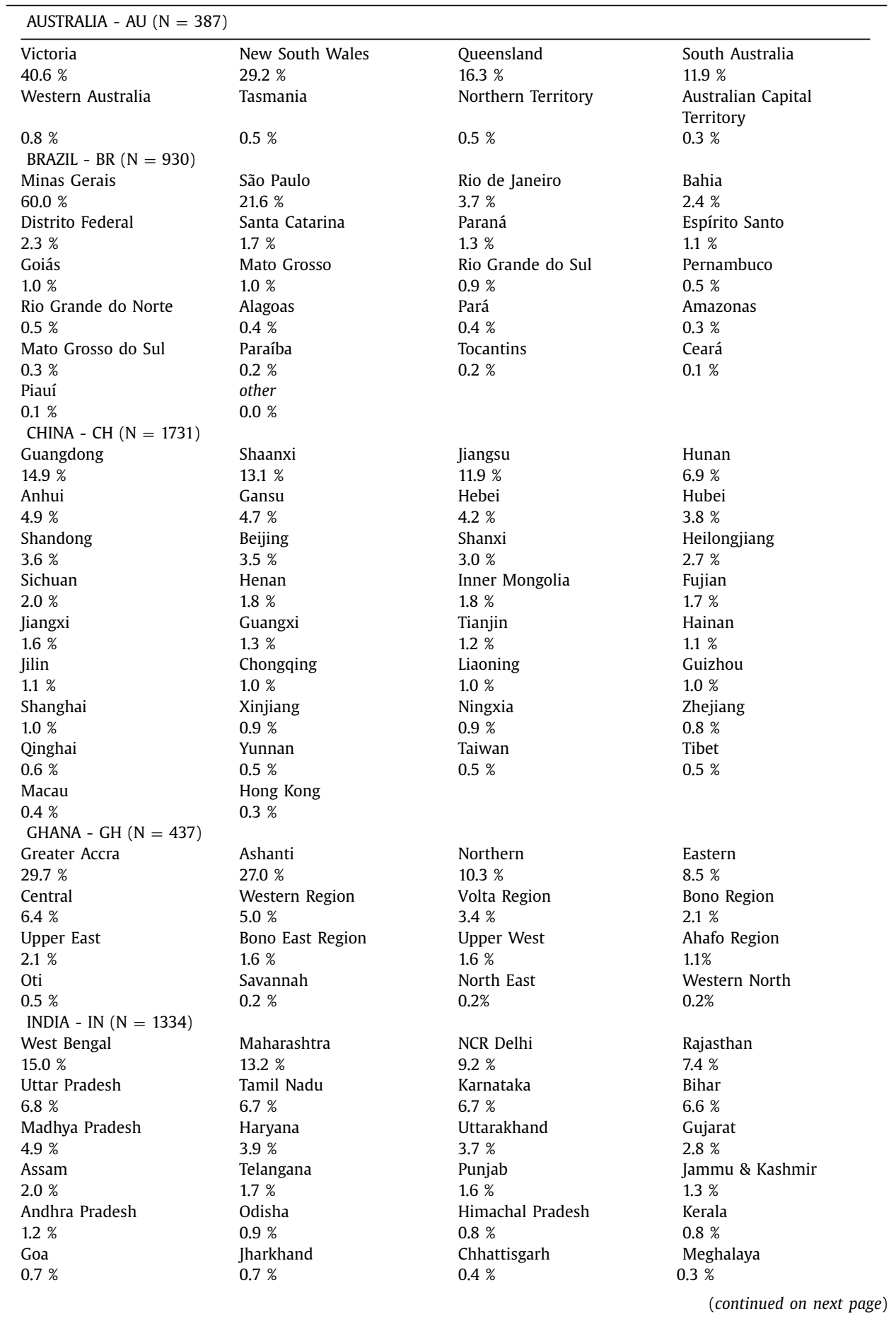


Table 1 (continued)

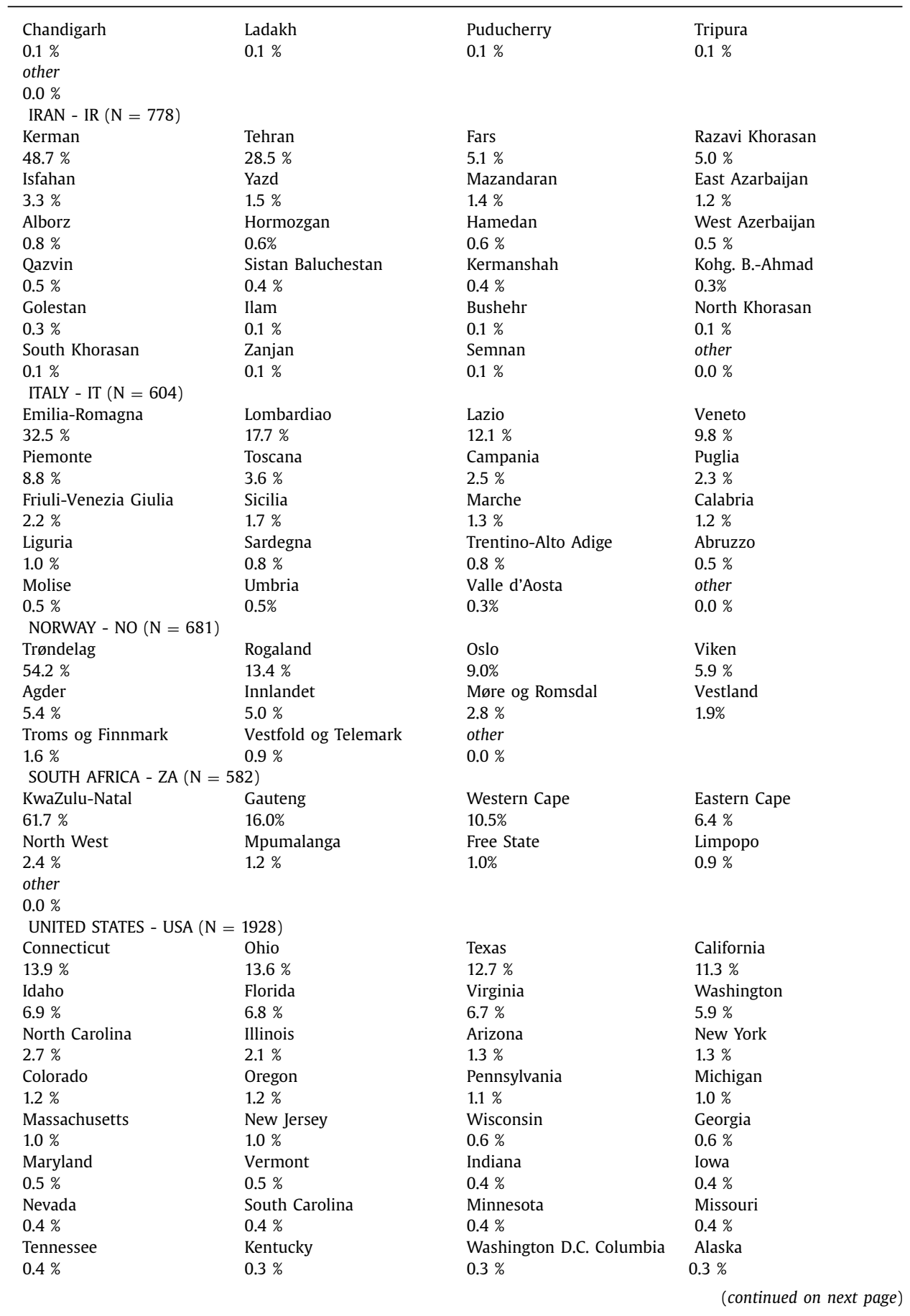


Table 1 (continued)

\begin{tabular}{llll}
\hline West Virginia & Alabama & Arkansas & Kansas \\
$0.3 \%$ & $0.2 \%$ & $0.2 \%$ & $0.2 \%$ \\
Louisiana & New Hampshire & Montana & North Dakota \\
$0.2 \%$ & $0.2 \%$ & $0.2 \%$ & $0.1 \%$ \\
Maine & Rhode Island & Wyoming & Hawaii \\
$0.1 \%$ & $0.1 \%$ & $0.1 \%$ & $0.1 \%$ \\
Nebraska & New Mexico & Oklahoma & South Dakota \\
$0.1 \%$ & $0.1 \%$ & $0.1 \%$ & $0.1 \%$ \\
Utah & Guam & US Virgin Islands & other \\
$0.1 \%$ & $0.1 \%$ & $0.1 \%$ & $0.0 \%$ \\
\hline
\end{tabular}

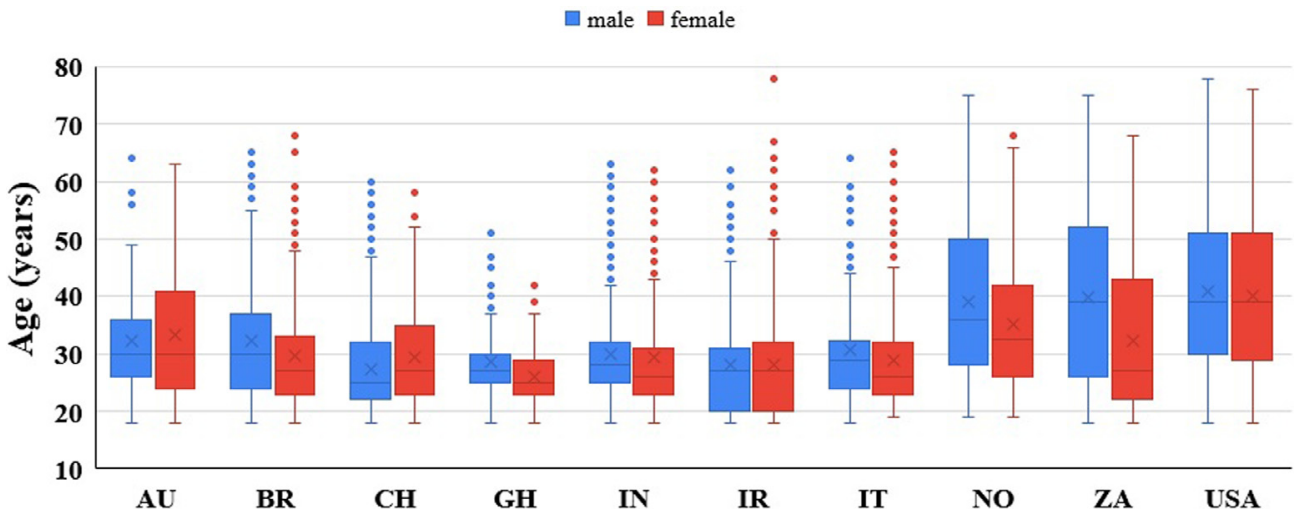

Fig. 1. Age and gender of the respondents for each country.

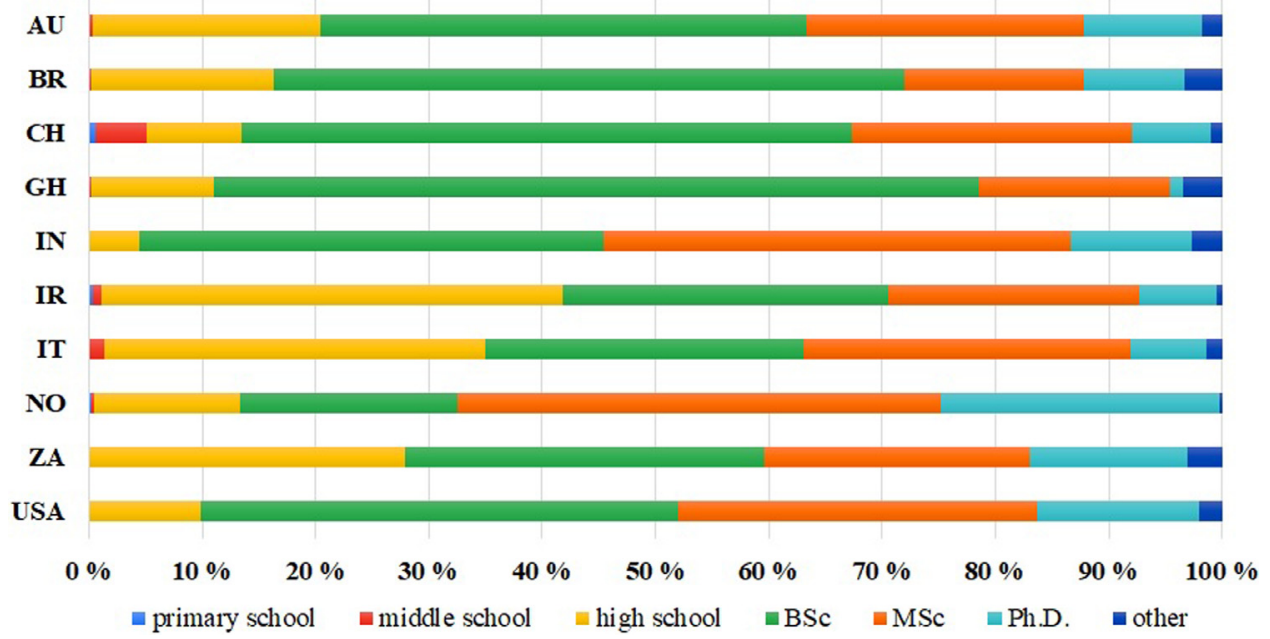

Fig. 2. Education of the respondents for each country.

\section{Experimental design, materials, and methods}

The online survey has assessed the air quality as subjectively perceived by citizens in ten countries: Australia, Brazil, China, Ghana, India, Iran, Italy, Norway, South Africa and the United States. The online questionnaire was hosted on two platforms: Google Forms (English, Italian, 


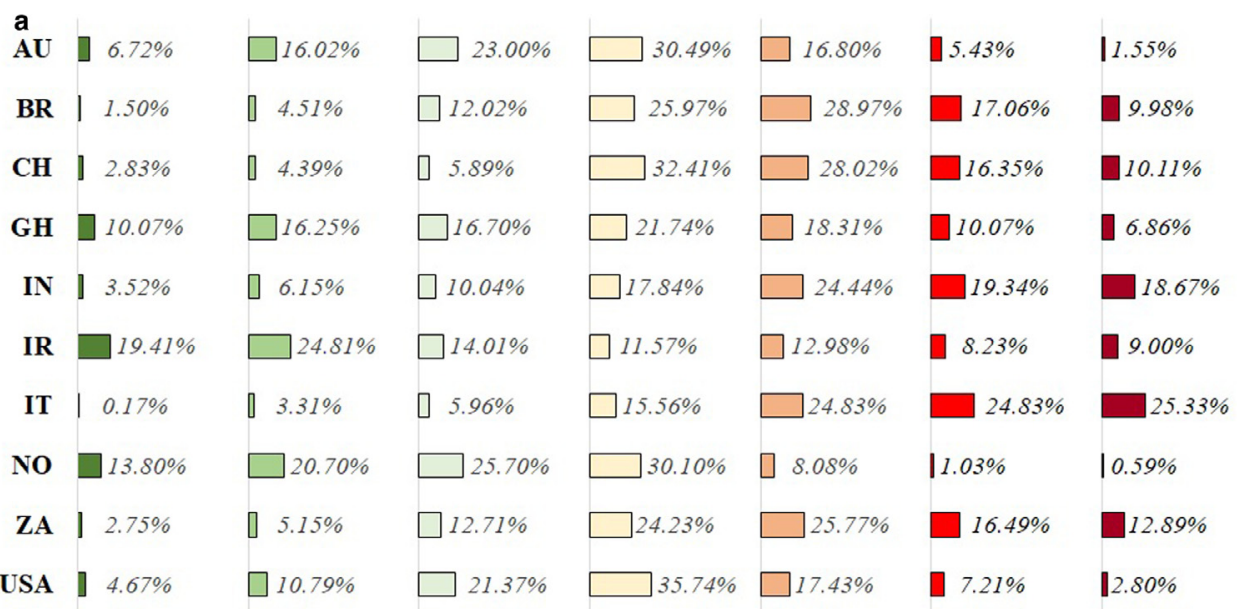

$\square$ extremely low/absent $\quad$ घvery low $\quad \square$ low $\square$ average $\quad \square$ high $\quad$ very high $\square$ extremely high

b

\begin{tabular}{|c|c|c|c|c|c|c|c|}
\hline $\mathbf{A U}$ & $23.26 \%$ & \begin{tabular}{|l}
$\square$ \\
\end{tabular} $1.27 \%$ & \begin{tabular}{|l}
$\square$ \\
\end{tabular} & $\square 14.47 \%$ & 』 $1.55 \%$ & $0.26 \%$ & $0.00 \%$ \\
\hline BR & $6.33 \%$ & $\square 15.02 \%$ & $\square$ 35.94\% & $\square 29.83 \%$ & $\square 10.30 \%$ & | $2.36 \%$ & $0.21 \%$ \\
\hline $\mathrm{CH}$ & ] $4.79 \%$ & $5.08 \%$ & $\square 11.96 \%$ & $40.50 \%$ & $\square 18.72 \%$ & $\square 12.19 \%$ & $6.76 \%$ \\
\hline GH & $15.79 \%$ & $\square 24.49 \%$ & $24.94 \%$ & $\square 21.28 \%$ & $7.55 \%$ & $4.81 \%$ & $1.14 \%$ \\
\hline IN & $23.84 \%$ & \begin{tabular}{|l}
$\square$ \\
\end{tabular} $2.98 \%$ & $30.36 \%$ & $\square 10.42 \%$ & $1.42 \%$ & $0.45 \%$ & $0.52 \%$ \\
\hline IR & $40.49 \%$ & $26.86 \%$ & $\square 13.75 \%$ & $\square 11.95 \%$ & $4.50 \%$ & $1.54 \%$ & $0.90 \%$ \\
\hline IT & $10.26 \%$ & $\square 15.40 \%$ & $35.60 \%$ & \begin{tabular}{|l}
$\square$ \\
\end{tabular} & $\square 8.61 \%$ & 1 $1.99 \%$ & $0.50 \%$ \\
\hline NO & $21.44 \%$ & $\square 28.63 \%$ & $\begin{array}{l}\square 0.40 \% \\
\end{array}$ & $17.33 \%$ & | $1.62 \%$ & $0.44 \%$ & $0.15 \%$ \\
\hline $\mathbf{Z A}$ & $13.57 \%$ & $\square 25.26 \%$ & $33.16 \%$ & $\square 19.07 \%$ & $6.01 \%$ & | $1.89 \%$ & | $1.03 \%$ \\
\hline USA & $\square 13.23 \%$ & \begin{tabular}{|l}
$\square$ \\
\end{tabular} $26.76 \%$ & $35.27 \%$ & $\square 18.83 \%$ & || $3.22 \%$ & $1.66 \%$ & $1.04 \%$ \\
\hline
\end{tabular}

$\square$ extremely low/absent $\square$ very low $\quad$ low $\square$ average $\quad \square$ high $\quad$ very high $\quad$ aextremely high

Fig. 3. Perceived amount of air pollution before (a) and during (b) the COVID-19 restrictions as experienced by the survey respondents in each country.

Norwegian, Persian, Portuguese versions) and WenJuanXing (Chinese version) and promoted on professional and social networks. The survey content was the same for each language; only the question regarding the respondents' geographical location was tailored for each country. A Likert scale was employed to collect information about subjective perceptions [2] regarding both the situation before and during the enforcement of the restrictions due to the COVID-19 pandemic $[3,4]$. The online survey was distributed using a combination of purposive and snowball techniques between 11-05-2020 and 31-05-2020. Previously, other opinion surveys at regional and national scale also dealt with the perception of air quality [5-7] and examined the psychological impacts on people's subjective emotional state [8]. The created dataset can allow to explore how air quality was experienced by the populations dealing with different levels of air pollution before the COVID-19 outbreak [9-11]. 


\section{Ethics statement}

All the survey respondents informed their consent before joining the survey consistent with the Declaration of Helsinki.

\section{Credit Author Statement}

Diego Maria Barbieri

Conceptualization, Methodology, Formal analysis, Investigation, Resources, Data curation, Writing - Original Draft, Visualization, Project administration

Baowen Lou

Conceptualization, Methodology, Formal analysis, Investigation, Resources, Data curation, Writing - Original Draft, Visualization

Marco Passavanti

Conceptualization, Methodology, Investigation, Writing - Original Draft, Visualization

Cang Hui

Investigation, Data curation, Writing - Review \& Editing, Visualization, Supervision

Daniela Antunes Lessa

Investigation, Data curation

Brij Maharaj

Investigation, Data curation

Arunabha Banerjee

Investigation, Data curation

Fusong Wang

Investigation, Data curation

Kevin Chang

Investigation, Data curation

Bhaven Naik

Investigation, Data curation

Lei Yu

Investigation, Data curation

Zhuangzhuang Liu

Investigation, Data curation

Gaurav Sikka

Investigation, Data curation

Andrew Tucker

Investigation, Data curation

Ali Foroutan Mirhosseini

Investigation, Data curation

Sahra Naseri

Investigation, Data curation

Yaning Qiao

Investigation, Data curation

Akshay Gupta

Investigation, Data curation

Montasir Abbas

Investigation, Data curation

Kevin Fang

Investigation, Data curation

Navid Ghasemi

Investigation, Data curation

Prince Peprah 
Investigation, Data curation

Shubham Goswami

Investigation, Data curation

Amir Hessami

Investigation, Data curation

Nithin Agarwal

Investigation, Data curation

Louisa Lam

Investigation, Data curation

Solomon Adomako

Investigation, Data curation

\section{Declaration of competing interest}

This research has not received any specific grant from funding agencies in the public, commercial, or not-for-profit sectors.

\section{Acknowledgments}

The precious support kindly provided by the following academics, researchers and professionals has been greatly appreciated: Mr. Fabio Selva (Heilongjiang International University, China), Mr. Marius Tangerås (Norwegian National Railway Administration Bane NOR, Norway), Dr. Azadeh Lak (Shahid Beheshti University, Iran), Dr. Barbara Stolte Bezerra (Universidade Estadual Paulista, Brazil), Dr. Xiaolong Sun (Guangdong University of Technology, China), Dr. Kasun Wijayaratna (University of Technology Sydney, Australia), Dr. Abdul Rahaman (Bharathidasan University, India), Dr. Dok Yen David Mbabil (Tamale Technical University, Ghana), Mr. Smit Bharat Thakkar (Queensland University of Technology, Australia), Mr. Solomon Kwadwo Achinah (University of Cape Coast, Ghana), Dr. Olaf Weyl (South African Institute for Aquatic Biodiversity, South Africa), Mr. Ayush Dhankute (Atkins Ltd., India), Mr. Mohammadreza Zare Reisabadi (University of Adelaide, Australia), Dr. Sachin Gunthe (Indian Institute of Technology Madras, India), Dr. Issam Qamhia (University of Illinois at Urbana-Champaign, United States), Dr. Parama Bannerji (West Bengal College, India), Mr. Amirhosein Mousavi (University of Southern California, United States), Mr. Anshu Bamney (Rewa Engineering College, India), Dr. Yuefeng Zhu (Shijiazhuang Tiedao University, China), Dr. Jorge Ubirajara Pedreira Junior (Federal University of Bahia, Brazil), Dr. Andrea Colagrossi (Politenico di Milano, Italy) and Dr. Akhilesh Kumar Maurya (Indian Institute of Technology Guwahati, India).

\section{Supplementary materials}

Supplementary material associated with this article can be found, in the online version, at doi:10.1016/j.dib.2020.106169.

\section{References}

[1] D.M. Barbieri, B. Lou, M. Passavanti, C. Hui, D.A. Lessa, B. Maharaj, A. Banerjee, F. Wang, K. Chang, B. Naik, L. Yu, Z. Liu, G. Sikka, A. Tucker, A. Foroutan Mirhosseini, S. Naseri, Y. Qiao, A. Gupta, M. Abbas, K. Fang, N. Ghasemi, P. Peprah, S. Goswami, A. Hessami, N. Agarwal, L. Lam, S. Adomako, Perceived air pollution in Australia, Brazil, China, Ghana, India, Iran, Italy, Norway, South Africa, USA before and during COVID-19 restrictions, (2020). http: //dx.doi.org/10.17632/fb38h4tyzn.2.

[2] D. Stockemer, Quantitative Methods for the Social Sciences, Springer, Cham, 2019 https://doi.org/10.1007/ 978-3-319-99118-4. 
[3] T. Acter, N. Uddin, J. Das, A. Akhter, T.R. Choudhury, S. Kim, Evolution of severe acute respiratory syndrome coronavirus 2 (SARS-CoV-2) as coronavirus disease 2019 (COVID-19) pandemic: A global health emergency, Sci. Total Environ. 730 (2020) 138996, doi:10.1016/j.scitotenv.2020.138996.

[4] Oxford University, Coronavirus government response tracker, (2020). https://www.bsg.ox.ac.uk/research/ research-projects/coronavirus-government-response-tracker (accessed June 30, 2020).

[5] M. Nikolopoulou, J. Kleissl, P.F. Linden, S. Lykoudis, Pedestrians' perception of environmental stimuli through field surveys: Focus on particulate pollution, Sci. Total Environ. 409 (2011) 2493-2502, doi:10.1016/j.scitotenv.2011.02. 002.

[6] T.G. Reames, M.A. Bravo, People, place and pollution: Investigating relationships between air quality perceptions, health concerns, exposure, and individual- and area-level characteristics, Environ. Int. 122 (2019) 244-255, doi:10. 1016/j.envint.2018.11.013.

[7] S. Pu, Z. Shao, M. Fang, L. Yang, R. Liu, J. Bi, Z. Ma, Spatial distribution of the public's risk perception for air pollution: a nationwide study in China, Sci. Total Environ. 655 (2019) 454-462, doi:10.1016/j.scitotenv.2018.11.232.

[8] Y. Li, D. Guan, S. Tao, X. Wang, K. He, A review of air pollution impact on subjective well-being: Survey versus visual psychophysics, J. Clean. Prod. 184 (2018) 959-968, doi:10.1016/j.jclepro.2018.02.296.

[9] P. Lal, A. Kumar, S. Kumar, S. Kumari, P. Saikia, A. Dayanandan, D. Adhikari, M.L. Khan, The dark cloud with a silver lining: Assessing the impact of the SARS COVID-19 pandemic on the global environment, Sci. Total Environ. 732 (2020) 139297, doi:10.1016/j.scitotenv.2020.139297.

[10] S. Muhammad, X. Long, M. Salman, COVID-19 pandemic and environmental pollution: A blessing in disguise? Sci. Total Environ. 728 (2020) 138820, doi:10.1016/j.scitotenv.2020.138820.

[11] A. Chauhan, R.P. Singh, Decline in PM2.5 concentrations over major cities around the world associated with COVID19, Environ. Res. 187 (2020) 109634, doi:10.1016/j.envres.2020.109634. 